\title{
Reflets
}

Revue ontaroise d'intervention sociale et communautaire

\section{Le secret et l'enfant victime de maltraitance sexuelle}

\section{Martine de Lajudie}

Volume 2, numéro 1, printemps 1996

Contrer la violence subie par les femmes et les enfants en milieu familial

URI : https://id.erudit.org/iderudit/026106ar

DOI : https://doi.org/10.7202/026106ar

Aller au sommaire du numéro

Éditeur(s)

Reflets : Revue ontaroise d'intervention sociale et communautaire

ISSN

1203-4576 (imprimé)

1712-8498 (numérique)

Découvrir la revue

Citer cet article

de Lajudie, M. (1996). Le secret et l'enfant victime de maltraitance sexuelle. Reflets, 2(1), 69-95. https://doi.org/10.7202/026106ar

\section{Résumé de l'article}

Cet article met en évidence la mutiplicité des raisons qui poussent un enfant à garder le silence sur sa victimisation sexuelle. Trois catégories de facteurs ont été identifiées. La première se rapporte au stade de développement de l'enfant. La seconde concerne l'agresseur sexuel et les épisodes de maltraitance sexuelle. La troisième relève des caractéristiques du milieu de soutien de l'enfant et de la façon dont elles sont perçues par ce dernier.
Tous droits réservés (C) Reflets : Revue ontaroise d'intervention sociale et communautaire, 1996
Ce document est protégé par la loi sur le droit d'auteur. L'utilisation des services d'Érudit (y compris la reproduction) est assujettie à sa politique d'utilisation que vous pouvez consulter en ligne.

https://apropos.erudit.org/fr/usagers/politique-dutilisation/ 


\section{Le secret et l'enfant victime de maltraitance sexuelle}

C et artide met en évidence la mutiplicité des raisons qui poussent un enfant à garder le silence sur sa vidimisation sexuelle Trois catégories de facteurs ont été identifiées $L$ a première se rapporte au stade de développement de l'enfant. L a seconde concerne l'agresseur sexuel et les épisodes de maltraitance sexuelle $L$ a troisième relève des caractéristiques du milieu de soutien de l'enfant et de la façon dont elles sont pergues par ce dernier.

\section{M artine de $L$ ajudie}

D épartement de psychologie, U niversité L aurentienne

\section{Introduction}

La maltraitance sexuelle des enfants est un phénomène largement répandu, une réalité de plus en plus reconnue ${ }^{1}$ et ce, de façon primordiale, et dont la perception a changé considérablement (Frude, 1982). De nombreux facteurs d'ordre historique, psychologique et politique, ainsi que l'ignorance dansce domaine, expliquent l'arrivée relativement récente du sujet à l'avant-scène des problèmes sociaux. L'un d'entre eux est le secret dans lequel certaines victimes gardent leurs expériences traumatisantes (Farrell, 1988; G elinas, 1983; Sorensen, 1991). II est évident qu'on ne peut contrer cette violence que constitue la maltraitance sexuelle tant que l'on n'en connaît pas l'existence.

Les victimes de maltraitance sexuelle se répartissent tout le long d'un continuum du secret, depuis celles qui révèlent leurs expériences immédiatement et totalement, à celles qui gardent le secret. II est difficile de connaitre le pourcentage d'enfants qui dévoilent immédiatement leur maltraitance, celle-ci n'étant alors souvent pas ébruitée hors de l'entourage immédiat. C e que l'on sait 
«C ertains enfants ne parlent jamais de leur exploitation sexuelle... Le secret peut alors être gardé jusqu'à l'âge adulte, et même indéfiniment.» par contre, c'est que le nombre d'enfants qui ne parlent pas est grand, les victimes ne parlant souvent qu'à l'âge adulte. De plus, ce nombre augmente avec la gravité des cas (Farrell, 1988). Certains enfants ne parlent jamais de leur exploitation sexuelle. C'est souvent le cas dans les situations d'inceste (Farrell, 1988). Le secret peut alors être gardé jusqu'à l'âge adulte, et même indéfiniment.

Le secret fait partie du même type de phénomènes que la révélation. Tous deux sont de l'ordre de la communication. U n enfant qui parle de sa victimisation sexuelle est celui pour lequel lesfacteurs poussant au secret ne jouent pas ou plus. M ême lorsque l'enfant ne parle pas d'emblée de sa maltraitance sexuelle, il y a une multitude de facteurs qui peuvent l'inciter à abandonner son silence par la suite. $0 \mathrm{n}$ peut prendre pour exemple les programmes d'éducation sur la maltraitance sexuelle. Ils sont parfois accompagnés d'une prise de conscience par l'enfant de la nature anormale de ses expériences et des responsabilités respectives des protagonistes. D e tels programmes peuvent le conduire à parler à ses éducateurs ( $G$ entles et C assidy, 1991; Ferguson et M endelsonAges, 1991). O u encore, certains enfants ne font de révélations qu'en dehors de leur famille. Lorsque l'enfant parle à ses camarades, ceux-ci, dans quelques cas, vont informer un adulte. Le secret, en conséquence, peut être temporaire ou permanent. Les deux types de secret se retrouvent chez nos sujets. Certains enfantsne parlent d'abord pas de leur victimisation et ne la révèlent que plustard. $0 \mathrm{n}$ peut alors étudier rétrospectivement les raisons de leur silence originel. Les autres enfants refusent de parler de leur maltraitance sexuelle qui n'est donc connue que grâce à des sources externes à l'enfant (témoins, autres victimes, examens médicaux, et plus souvent encore révélation par inadvertance chez les enfants les plus jeunes).

$\mathrm{N}$ otre travail consiste à présenter quelques-unes des très nombreuses raisons qui peuvent pousser un enfant à garder le silence. Le but d'une telle présentation est de décrire cette réalité du silence des victimes de maltraitance sexuelle, et d'en montrer la diversité et la complexité. Par conséquent, la liste qui est donnée, des facteurs et des motivations qui portent au secret, ne doit pas être vue comme exhaustive. Par ailleurs, les motivations et les 
facteurs mentionnés dérivent de l'analyse des cas tirés de la population à l'étude, telle qu'elle est décrite ci-dessous. Ils ne proviennent donc directement ni de l'étude de la littérature, ni de modèles théoriques.

\section{Sujets et méthode}

Les sujets qui ont servi de base pour cette étude proviennent du $\mathrm{N}$ ord-est de l'O ntario. II s'agit d'un échantillon de 69 enfants ou adolescentes ${ }^{2}$, tiré d'une population plus large de 300 sujets, et sélectionné parce que la maltraitance sexuelle était l'une des composantes de la problématique du sujet.

Ces enfants ont été vus dans le cadre de consultations psychologiques, pour une évaluation ou pour une psychothérapie. Pour la plupart, ils ont été envoyés ou suivis par la Société d'aide à l'enfance locale (62 soit $90 \%$ ). Les 7 autres ont été vus dans le milieu scolaire. Les techniques d'évaluation et de collecte des données ont été à peu près similaires d'un enfant à l'autre. Les cas de maltraitance sexuelle ont presque tous donné lieu à une enquête sociale (61/ 69 soit $88 \%$ ) qui avait commencé avant la consultation dans la majorité des cas (58/61 soit $90 \%)$. Vingt-huit enfants sur 69 (41\%) étaient en foyer nourricier au moment des consultations II y avait 45 filles (65 \%) et 24 garçons (35\%), 36 francophones (51 \%), 25 anglophones (36\%) et 8 A mérindiens (12\%).

Tableau 1 - Tableau des âges par sexe

\begin{tabular}{lcc}
\hline & Garçons & Filles \\
M oins de 4 ans & 5 & 4 \\
4 et 5 ans & 8 & 7 \\
6,7 et 8 ans & 2 & 8 \\
$9,10,11,12$ ans & 2 & 11 \\
13 et 14 ans & 2 & 6 \\
plus de 14 ans & 5 & 9 \\
\hline Total des cas: 69 & 24 & 45
\end{tabular}


«C omme les études statistiques l'indiquent constamment, peu de sujets ont été victimes de personnes étrangères au milieu familial.»
$D$ ans beaucoup de cas, la maltraitance sexuelle subie était sévère, avec viol et/ ou violence physique (43/69 soit $62 \%)$. Le tiers (32\%) des sujets était des victimes d'inceste de la part d'une figure parentale. Comme les études statistiques l'indiquent constamment, peu de sujets ont été victimes de personnes étrangères au milieu familial. U ne seule enfant dans notre population a été agressée par un inconnu.

II faut considérer notre recherche comme une étude préliminaire tentant de dégager des catégories d'analyse pouvant orienter la recherche future plutôt, que comme présentant un modèle définitif. En effet, nos données, comme nous venons de le dire, sont de type archival. Par conséquent, l'échantillon sélectionné ne peut être rapporté à une population parente précise. De plus, les données n'ont pas été recueillies de façon systématique puisque, lorsqu'elles ont été rassemblées, l'objet de l'étude n'était pasencore connu. C'est la raison pour laquelle nous ne présentons pas de tableaux ou n'effectuons pas de calculs statistiques pour tenter de classer les sujets en fonction des facteurs que nous avons isolés.

Le matériel recueilli, par sa richesse de vécu, permet d'en effectuer l'étude phénoménologique (D enzin 1986, 1989). C elleci consiste à prendre pour ce qu'elles sont les productions des sujets (plutôt que d'analyser les données à partir d'hypothèses). «La psychologie phénoménologique [est une] tentative de décrire les essences des expériences) (K lein et Westcott, 1994). C ette méthode a déjà été utilisée pour la maltraitance sexuelle, généralement auprès d'adultes (M cEvoy et $D$ aniluk, 1995) plutôt que d'enfants ( $D$ aldin, 1988). D Dans notre étude, les propos des enfants qui se rapportaient au secret ont été isolés. U ne tentative de compréhension de ces proposa été entreprise en les replaçant dans l'ensemble du cas. U n regroupement a permis d'aboutir à notre liste de facteurs. $\mathrm{N}$ ous avons dans la mesure du possible utilisé des exemples comme illustrations.

Deux principes sont à la base de l'essai de compréhension que constitue cette étude. Le premier est que l'on tient compte du niveau de développement psychologique de l'enfant. La pensée du jeune enfant obéit à ses propres règles et il faut les connaître pour comprendre son discours. Par exemple, le même mot sexuel 
n'a pas la même signification dans la bouche d'un enfant de 5 ans et dans celle d'un adolescent. Le second principe est celui de l'importance d'envisager l'enfant avec ses pensées et ses émotions comme un tout. C haque expérience vécue est intégrée dans une certaine vision du monde pré-existante et la modifie à son tour. $C$ 'est ainsi que, placés dans des circonstances semblables, les enfants vont réagir différemment.

\section{Révélation spontanée et immédiate de l'enfant}

Q uelques victimes révèlent spontanément et immédiatement la maltraitance sexuelle qu'elles ont subie. Les facteurs suivants semblent favoriser ces déclarations spontanées:

- la capacité de l'enfant à s'exprimer. C ette capacité inclut le langage non-verbal, et suppose, de la part de l'enfant, une intuition et une pratique de ce qu'est la communication.Toutefois, les enfants négligés accusent généralement un déficit dans ce domaine (Barahal et al., 1981; Cicchetti et Beeghly, 1987).

- la propension de l'enfant à parler. U n enfant bavard et ouvert seraplusenclin à raconter sesexpériencesquellesqu'elles soient.

- l'innocence de l'enfant dans le domaine sexuel. L'enfant n'ayant pas encore conscience de ce qui est socialement acceptable ou inacceptable ne sanctionne pas son discours. II va donc utiliser un langage direct et concret, et avoir moins d'inhibitions.

- la disponibilité des parents vis-à-vis de ce que l'enfant a à dire, et leur capacité à l'aider à s'exprimer et à le comprendre. Bien des parents sont trop surchargés ou préoccupés pour prendre le temps de faire parler et d'écouter leur enfant, ou encore ils ont l'opinion sous-jacente que ce que dit l'enfant est sans signification importante. C es parents ne vont pas être attentifsaux détailsinhabituels du discours de l'enfant (Belsky et Vondra, 1987). 
- le contact affectif de l'enfant avec ses parents. Ce facteur est lié à deux des facteurs prédédents. En effet, le contact affectif de bonne qualité entre un enfant et ses parents est essentiel pour que l'enfant développe sa capacité à s'exprimer (premier facteur). Par ailleurs, la disponibilité des parents vis-à-vis de leur enfant (quatrième facteur) est l'une des composantes du contact affectif de l'enfant avec ses parents: un enfant se sentira d'autant mieux compris que ses parents seront plus disponibles à son égard.

- la forte valence affective soit positive, soit négative de ce qui a été vécu. Plus l'expérience de maltraitance sexuelle aura été marquante (et cela ne veut pas forcément dire grave du point de vue de l'adulte), plus l'enfant se sentira poussé à la partager.

- la croyance de l'enfant selon laquelle la maltraitance sexuelle va stopper s'il parle (dans les cas d'expériences désagréables).

\section{G érard 3 : «manger pi pi- G érard» 4}

$G$ érard ( 2 ans et demi) est placé chaque jour dans un foyer pendant que ses parents travaillent. Le soir, en retrouvant ceux-ci, il raconte avec force détails ce qui s'est passé dans la journée et explique comme il s'est amusé. À deux reprises à son père interloqué, il dé dare: «(nom du gardien) manger pipi-G érard».

II raconte aussi que, dans ces circonstances, le gardien se sauve avant que sa femme n'arrive.

Tous les facteurs énumérés plus haut, sauf le dernier, se trouvent réunis dans le cas de cet enfant. II s'exprimait suffisamment bien; il était bavard, et innocent dans le domaine sex uel. II avait un bon contad avec son père II bénéficiait d'un père attentif qui, d'une

«... ayant trouvé

l'expérience non traumatisante, l'enfant a désiré en faire part à son père.» part, a écouté son enfant et, d'autre part, a réalisé la portée de ce que son fils lui disait. $D$ e plus ce père n'a pas réagi d'une façon qui aurait pu effrayer l' enfant et ainsi fausser ou arrêter sa dédaration. Finalement, ayant trouvé l'expérience non traumatisante, l'enfant a désiré en faire part à son père. 


\section{Le secret et ses raisons}

A près cette brève présentation desfacteurs qui peuvent entrer en jeu dans les cas où l'enfant révèle sa maltraitance sexuelle, nous allonsmaintenant présenter quelques-unes des raisonsqui peuvent pousser un enfant à garder le secret au sujet de ses expériences de maltraitance sexuelle. C esraisonsont pu être élucidées, soit parce que l'enfant les a explicitées par la suite, soit par recoupements ou interprétation des données. C ependant, dans de nombreux cas, on ne connaît pas de façon certaine la cause du secret. 0 n ne peut que faire des hypothèses. C 'est ce qui se passe, en particulier lorsque l'enfant refuse de parler de ses expériences de maltraitance sexuelle, même après que celles-ci ont été découvertes.

O n peut, de façon un peu schématique il est vrai, catégoriser les raisons du maintien du secret par l'enfant en trois classes: il y a d'abord cellesqui se rapportent àl'enfant lui-même; il y a ensuite celles qui sont liées aux caractéristiques des épisodes de maltraitance sexuelle àl'agresseur ou de ce qui y touche (comme la sexualité); enfin, il y a celles qui concernent le système de soutien de l'enfant.

\section{Raisons du silence propres à l'enfant}

«C ertaines caractéristiques de la mentalité enfantine,... peuvent le conduire à garder le silence... »
U ne raison expliquant le maintien du secret peut être définie comme étant propre à l'enfant lorsqu'on peut la comprendre en considérant le niveau de développement, la personnalité ou les expériences antérieures de la victime.

\section{Secret et stade de développement}

C ertaines caractéristiques de la mentalité enfantine, telles qu'elles existent à divers stades de son développement, peuvent le conduire à garder le silence de la même façon qu'elles peuvent 
«... certaines vidimes d'inceste peuvent, pendant des années en vouloir à leur mère de ne pas les avoir protégées, alors que ce sont elles qui ont gardé le silence» le conduire à parler (cf. naïveté dans le domaine sexuel).

Pendant la petite enfance, l'enfant ne se distingue pas clairement de sa mère psychologiquement. C'est la période symbiotique. Par la suite, il sait être une personne distincte, maisil croit que ses parents sont omniscients et extralucides. II pense que ceux-ci savent ce qui lui arrive et ce qu'il pense. Par conséquent, il n'éprouve pas le besoin de parler de choses qu'il pense être déjà connues. À un degré de développement un peu plus avancé, il lui semble suffisant de parler d'une partie d'une situation pour que le parent en connaisse l'ensemble.

D e telles croyances peuvent avoir des conséquences dramatiques à long terme lorsqu'elles ne sont pas révisées au cours de la vie (Jehu et al., 1986). Par exemple, certaines victimes d'inceste peuvent, pendant des années, en vouloir à leur mère de ne pas les avoir protégées, alors que ce sont elles qui ont gardé le silence.

\section{A manda: sa mère «laissait faire »}

\begin{abstract}
A 8 ans A manda était vicime de relations incestueuses $E$ lle gardait son secret. E lle présentait des troubles du comportement, étant colérique et agressive Sa mère lui demanda un jour la raison de cet état continuel de frustration. Sa fillelui répondit qu' elle se sentait «sale», "comme une guenille»; mais elle ne parla pas de la cause de son état. E lle expliqua quelques années plus tard qu'en donnant sa réponse, elle s'était demandé pouquoi sa mère «laissait faire.»

L a contradicion apparente qui existe entre le secret que garde l'enfant et son désir d' être comprise disparaît lorsqu' on fait appel à la croyance enfantine en I'omniscience des parents A manda était convaincue que sa mère «laissait faire» puisqu' elle savait. E lle ne répondit pas vraiment à la question de sa mère
\end{abstract}

Lorsque l'enfant, au cours de son développement, découvre que ses parents ne savent pas tout et ne peuvent lire ses pensées, il accorde une grande importance au secret. II découvre qu'il peut choisir ce qu'il veut ou ne veut pas dire. Par conséquent, quand 
«L es phénomènes de dissociation, qui consistent à dissocier certains souvenirs du reste de la vie mentale, sont des processus de survie fréquemment utilisés» un enfant montre une certaine préoccupation pour le secret, on peut en déduire qu'il a quelque chose d'important à cacher, comme des épisodes de maltraitance sexuelle.

\section{Oubli et dissociation}

Lorsqu'il n'y a pas de révélation immédiate, les épisodes de maltraitance sexuelle peuvent être conservés en mémoire mais gardés secrets. Ils peuvent aussi être oubliés. Dans ce cas, les souvenirs ne sont plus disponibles au sujet en raison de facteurs qui peuvent être soit physiologiques, soit psychologiques (Brady, 1979). Les phénomènes de dissociation, qui consistent à dissocier certains souvenirs du reste de la vie mentale, sont des processus de survie fréquemment utilisés. Ils ont été particulièrement étudiés chez les victimes de maltraitance sexuelle une fois adultes (Bowman et al., 1985; Chu et Dill, 1990; Loftus, 1993). Les souvenirs peuvent redevenir conscients au cours de la vie, pour toutes sortes de raisons. La maturation sexuelle et les situations qui présentent des similarités avec la situation traumatique d'origine semblent jouer un rôle important. Finalement, les épisodes de maltraitance sexuelle peuvent ne pas avoir été enregistrés. On dit qu'ils n'ont pas été fixés. Ils ne peuvent, dans ces cas, être rappelés. On se trouve alors en présence d'un phénomène que l'on rencontre dans les cas de syndrome traumatique.

Les facteurs qui président à la capacité de rappel, à l'oubli ou à la non-fixation restent peu clairs dans les cas de maltraitance sexuelle, et font l'objet de vives controverses. Ce problème cependant concerne plus les adultes que les enfants.

\section{B ernadette: fixation partielle en mémoire?}

B ernadette ( 6 ans) a été violée par un gardien masaulin. E lle n'a rien révélé à sa mère, mais en a parlé à son institutrice, puis aux travailleuses sodales venues I' interroger par la suite Pendant la thérapie par le jeu, elle hésite à parler ouvertement de son traumatisme, mais elle le joued' une manière à peine déguisée $M$ algré cela, il reste impossible de définir la nature exacte du 
viol. $0 \mathrm{n}$ a l'impression, en observant son jeu en thé rapie, que l'enfant ne se souvient pas des parties les plus traumatisantes

C e dont B ernadette se souvient, c'est œ qui a précédé ou suivi les agressions: les circonstances, les lieux et le protagoniste E lle n'est pas capable de décire l'agression elle même. $0 \mathrm{n}$ ne sait - et les trois hypothèses doivent être faites - s'il s'agit d'un refoulement (d'un oubli de nature émotionnelle), d'un véritable oubli (à base physiologique, ayant eu lieu au moment ou juste après l'agression), ou si la partie traumatique n'a pas été fixée en mémoire.

\section{C aroline: sentiment d'incrédulité}

À l'âge de 6 ans, $C$ aroline a été vicime demaltraitance sexuelle de la plus grande sévérité de la part de plusieurs agresseurs, masalins et féminins. E lle a par la suite été adoptée dans un bon foyer. À 14 ans, elle ne se souvient de rien et a un sentiment d' incrédulité lorsqu' elle entend sa mère adoptive rapporter ce qu' elle disait huit ans plus tôt. E lle vient cependant en thérapie pour des épisodes dissodaatifs perturbateurs pendant lesquels elle entend des voix, ou voit des scènes de son passé.

$D$ ans ce cas, on peut voir qu'il y a eu dissociation. $C$ ette dernière a fait partie du processus thérapeutique qui a commencé lorsquel'enfant a étéadoptée À l'adolescence, lorsque la sexualité acquiert de l'importance, les souvenirs commencent à revenir, sous forme dissociée d'abord, plus daire par la suite.

\section{Protection de la vie intime et pudeur}

Losrqu'au cours de son développement, l'enfant prend conscience de ce qu'est le secret, il se fabrique une vie privée. De plus en plus, il va protéger celle-ci des regards indiscrets. 
L'adolescent se caractérise, entre autres, par l'importance de sa vie privée. $\mathrm{N}$ aturellement, il ne va donc pas parler spontanément à ses parents, ou même à qui que ce soit, de ses expériences de maltraitance sexuelle, indépendamment de la qualité de son système de soutien.

\section{A ndré}

À 8 ans, A ndré s'est fait violemment sodomiser. Plus tard, sa soeur raconte qu' au moment des événements, alors qu'elle avait 4 ans: "A ndré a dit de ne pas en parler. II m'a dit qu'il pensait qu'il avait été violé, mais que c'était un secret.

- Violé, c'est le mot qu'il a utilisé?

- 0 ui.»

Le secret ne deviendra public que 8 ans plus tard. $D$ ans les paroles du petit garçon, on peut voir sa tentative de définir la nature de l'agression qu'il a subie. Pourquoi ne voulait-il pas ébruiter la chose, on ne le sait pas vraiment. $0 \mathrm{n}$ peut voir la pudeur comme une explication possible du secret, plutôt que la peur, l'enfant n'ayant plus été en contact avec son agresseur depuis de nombreuses années

«L'attribution de responsabilité est de I'ordre des faits;' ... La aulpabilité dérive d'un sentiment de responsabilité morale... »

\section{Sentiment de responsabilité}

II y a une différence essentielle entre l'attribution de responsabilité et le sentiment de culpabilité. 0 n peut être la cause d'une catastrophe sans en être moralement responsable, donc sans qu'il soit justifié d'en être culpabilisé. L'attribution de responsabilité est de l'ordre des faits; elle repose sur une analyse objective (qui peut être erronée) de la situation. La culpabilité dérive d'un sentiment de responsabilité morale, c'est-à-dire d'un jugement de valeur selon lequel la personne a choisi volontairement l'action dont elle se sent coupable.

D ans le cas des enfants, plus encore que dans celui des adultes, il faut distinguer le jugement de l'enfant de celui de l'observateur, car ils peuvent différer grandement. Par le simple fait qu'il s'est 
trouvé impliqué dansun épisode de maltraitance sexuelle ou qu'il a attiré sur lui l'attention de l'agresseur, l'enfant peut se sentir responsable jusqu'à un certain point. D ans la majorité des cas, du point de vue adulte, ce sentiment de responsabilité de l'enfant peut paraitre injustifié. C'est pourtant un sentiment naturel et presqu'automatique chez les enfants, comme ce le serait chez les adultes. L'individu a un tel besoin de s'assumer qu'il préfère se croire responsable, plutôt que de se voir comme une victime impuissante. S'ajoute à cela une faute de jugement a posteriori; la personne pense qu'elle n'aurait pas dû se mettre dans certaines situations, sans tenir compte du fait que, si elle s'y est mise, c'est justement parce qu'elle ne savait pas ce qui pouvait arriver.

Plus encore que les petits enfants, pour des raisons développementales, les adolescentes font la différence entre leur responsabilité et celle de leur agresseur. Par conséquent, il est rare qu'une adolescente agressée sexuellement s'attribue la responsabilité morale de l'agression. Elle ne pense pas qu'elle a été agressée parce que c'était, au fond, ce qu'elle désirait. C ela ne veut pas dire qu'elle ne puisse pas se sentir responsable: étant plus autonome que l'enfant, elle va s'attribuer la responsabilité de s'être mise imprudemment dansune situation dangereuse. A u minimum, elle s'accuse de naïveté, qu'elle nomme souvent stupidité. Elle commet alors l'erreur de jugement a posteriori que nous venons de mentionner.

En pratique, l'enfant ou l'adolescente vont fréquemment utiliser l'expression «de ma faute» pour désigner soit leur sentiment d'être responsable de ce qui leur est arrivé (en se trouvant là, par exemple), soit leur sentiment de responsabilité morale. Cette imprécision du langage peut créer des confusions.

\section{D ominique: «Je pensais que c'était de ma faute »}

E $n$ consultation, D ominique (9 ans) parle avec la psychologue des épisodes de maltraitance aux quels I'a soumise un oncle de 18 ans. La psychologue pose une question générale à propos de ces épisodes: «Q U'est-ce que tu as pensé?

- Q ue c'était méchant quoi qu'il a fait. 
- Tu en as parlé à quelqu' un?

- Pas la première fois.

- Q u' est-œ que tu as pensé sur toi?

- Je me sentais mal.

- Q u' est-ce que tu veux dire: tu te sentais mal?

- Je ne me sentais pas bien.

- Tu pensais que c' était la faute de qui?

- De moi.

- Pourquoi?

- Je ne sais pas.

- Pourquoi tu ne l'as pas dit?

- Parce que je pensais que c' était de ma faute.»

Sans raison objective, $D$ ominique se sentait donc responsable et, par conséquent, elle n'a pas parlé pendant un certain temps. $C$ ependant elle ne s'attribue pas et nes' est probablement jamais attribuéla responsabilité morale des adions de son onde. Elle $\mathrm{a}$, au contraire, toujours perçu les adions de clui-d comme «méchantes». II est probable que ce dont $D$ ominique se trouvait responsable, c'est de s'être trouvée là et de n'avoir pas empêché les choses plus vigoureusement.

«... il est rare que

I'enfant se sente coupable des actions de l'agresseur, car il fait assez tôt la différence entre ce qu'il a choisi de faire et ce qu' autrui lui impose»

\section{Sentiment de culpabilité}

La question de la culpabilité de l'enfant face à la maltraitance sexuelle qu'il a subie est complexe (Gomes-Schwartz, 1990). À l'encontre d'une conception répandue, il est rare que l'enfant se sente coupable des actions de l'agresseur, car il fait assez tôt la différence entre ce qu'il a choisi de faire et ce qu'autrui lui impose. Par conséquent, lorsque l'enfant est culpabilisé, ce n'est pas parce qu'il pense avoir fait ce qu'il n'a pas fait. Garder le secret par culpabilité est une cause du secret beaucoup moins importante qu'on ne le prétend actuellement.

N ous avons parlé précédemment du sentiment de responsabilité et de ses causes. C hez l'adulte, il ne suffit pas de se sentir responsable de quelque chose pour en être culpabilisé. II faut de plus s'attribuer une responsabilité morale. L'enfant, par contre, 
ne fait pas encore clairement cette distinction entre responsabilité et responsabilité morale.

Lorsque l'enfant se sent responsable, du moins en partie, de ce qui lui est arrivé, de la façon dont nous l'avons discuté au paragraphe précédent, il peut en être culpabilisé, bien que ce ne soit toutefois pas la règle. $0 \mathrm{n}$ peut résumer dans le tableau 2 suivant ce qui se passe dans l'esprit de l'enfant lorsqu'il se sent coupable, et mettre en parallèle les pensées de l'adulte à propos de la maltraitance sexuelle vécue par l'enfant.

Tableau 2

\begin{tabular}{lccc}
\hline & responsabilité & $\begin{array}{c}\text { responsabilité } \\
\text { morale }\end{array}$ & culpabilité \\
\hline enfant & j'étais là & j'ai laissé faire & j'aurais dû refuser \\
adulte & il n'a pas & il n'avait pas & il n'a pas à se \\
& choisi & le contrôle & sentir coupable \\
\hline
\end{tabular}

Le jeune enfant ne possède pas encore de système de valeurs morales abstraites et intériorisées. II peut se penser responsable d'actions mauvaises lorsqu'elles ont été définies comme telles préalablement par les adultes. C'est le cas si, d'une part, ce qui est sexuel appartient à cette liste de choses défendues, et d'autre part, dans la mesure seulement où l'enfant a participé pleinement aux actions, ou en a retiré du plaisir.

Q uant au fait que l'enfant ne rend souvent pas son agresseur moralement responsable de façon explicite, cela provient simplement de ce que l'enfant al'habitude d'obéir aux ordres des adultes sans se poser de questions sur le bien-fondé, sur la valeur morale de leurs actions.

\section{B ernard: «'ai fait quelque chose de mal.»}

Lors d'une première rencontre, la mère de B ernard (10 ans) raconte:

«U n jour, B ernard a dit: 
- F aut que je te parle

II s'est mis à pleurer. II a dit: J'ai fait quelque chose de mal. [nom de l'agresseur] a commencé à me toucher.»

L'enfant parle dairement de son sentiment de culpabilité. R emarquons cependant qu'il ne dit pas qu'il est responsable moralement (il ne dit pas qu'il a choisi l'activité). L'enfant se pense probablement coupable d'avoir participé, de ne pas avoir refusé de façon plus véhémente.

«L e sentiment de alpabilité des adolescentes est plus fréquent et aigu que celui des enfants. »

«... I'adolescente caint, si elle révèle son abus, d'être soumise à des contrôles encore plus sévères que ceux qui sont en place actuellement.»
Le sentiment de culpabilité des adolescentes est plus fréquent et aigu que celui des enfants. II a sa source dans les facteurs suivants. L'adolescente peut être culpabilisée en raison de son manque de jugement et de son imprévoyance. Elle peut aussi se sentir culpabilisée vis-à-vis de ses parents, qui l'avaient mise en garde et lui avaient interdit certaines activités, sorties, ou horaires.

Ce sentiment de culpabilité peut être la raison du secret. L'erreur que l'adolescente fait, en ne révélant pas l'incident à ses parents, est une erreur de proportions: être sortie de la maison la nuit par la fenêtre est mal, mais aux yeux de parents sains, le viol n'est pas une punition appropriée.

II est vrai que de constater que la prudence prévoyante des parents se voit justifiée de façon éclatante n'est pas fait pour plaire aux adolescentes, car cette attitude des parents pourrait être aussi valable dans d'autres domaines: travail scolaire, fréquentations, etc... L'adolescente pourrait y voir une mise en cause de son bon jugement et de sa sagesse. De plus, de façon souvent justifiée, l'adolescente craint, si elle révèle son abus, d'être soumise à des contrôles encore plus sévères que ceux qui sont en place actuellement. Elle craint aussi, de façon plus abstraite, la sollicitude inquiète de ses parents, la composante inquiète amplifiant ses propres craintes et sa peur du monde. 


\section{Raisons touchant la maltraitance, l'agresseur ou ce qui $s^{\prime} y$ rapporte}

«D ans des cas qui ne sont pas rares, l'enfant ne parle pas parce qu'il est attaché à l'agresseur.»
La deuxième classe de raisons pour lesquellesl'enfant peut garder le silence est probablement la plus importante. Elle est assez rarement considérée à sa juste valeur bien que son importance ait été mentionnée dès 1981 (H erman, 1981). Elle se rapporte aux épisodes de maltraitance sexuelle eux-mêmes, à l'agresseur, et aux circonstances associées. II s'agit de menaces, intimidation, chantage, séduction, promesses, cadeaux et ainsi de suite.

Comme l'enfant n'est pas capable d'avoir un recul suffisant par rapport à l'ensemble de la situation, il ne peut peser le pour et le contre du silence en connaissance de cause. II ne peut mettre d'un côté les risques qu'il prendrait s'il parlait, et de l'autre les bénéfices qu'il en retirerait. Beaucoup plus que l'adulte, il est enfermé dans le présent et le futur proche. Par exemple, il peut ne pas être capable d'envisager que, s'il garde le secret, la maltraitance sexuelle va se perpétuer. Par ailleurs, I'enfant n'a pas le sens des proportions. II peut, par exemple, accepter la maltraitance sexuelle pour ne pas perdre un certain privilège.

\section{Attachement préalable à l'agresseur}

L'agresseur sexuel est habituellement une personne qui connaît l'enfant. U n lien émotionnel positif véritable peut exister entre eux, au moins avant l'exploitation sexuelle. D ans d'autres cas, le pédophile va faire des travaux d'approche pour s'attirer lesfaveurs de l'enfant. C e dernier n'est pas en mesure de deviner la motivation véritable de son futur agresseur.

D ans des cas qui ne sont pas rares, l'enfant ne parle pas parce qu'il est attaché à l'agresseur. De plus, les épisodes peuvent être agréables pour l'enfant, surtout au début, soit en raison des gratifications et de l'attention dont il bénéficie, soit, plus rarement, sexuellement. Au bout d'un certain temps, même si l'enfant se détache émotionnellement de son agresseur ou trouve les activités désagréables, il se trouve pris dans un cercle d'habitudes et va continuer à garder le silence. 


\section{C harles: I'admiration}

C harles a 5 ans Son cousin de 15 ans l'a exploité sexuellement à plusieurs reprises $L$ a situation a été découverte lorsque l'enfant l'a révélée par inadvertance à sa mère, et aussi parce que d'autres victimes ont parlé. E $n$ effet, lors d'un bain, l'enfant a dit à sa mère: «(nom de l'agresseur), il touche mon zizi.»

$M$ algré cela, $C$ harles ne veut rien dire II y a plusieurs raisons à son silence L'une d'entre elles est la grande admiration que $\mathrm{C}$ harles porte à son cousin, et son désir de ne pas le mettre dans l'embarras

\section{Utilisation de moyens coercitifs par l'agresseur}

Toutes sortes de moyens peuvent être utilisés par l'agresseur sexuel pour obtenir le silence (et la coopération) de sa victime. Elles tendent à créer un état de peur chez l'enfant, et sont probablement la cause la plus fréquente de son silence. Leur importance capitale est rarement reconnue, et le grand public en minimise l'effet (Broussard et Wagner, 1988; D ell, 1989). Lorsqu'il se sent menacé, il est rare que l'enfant parle. Parmi les moyens utilisés par les agresseurs sexuels, on peut citer la séduction, les méthodes éducatives (l'agresseur expliquant à l'enfant qu'il doit l'éduquer dans le domaine sexuel, par exemple), le mensonge, l'appel à la compréhension, à la pitié, l'achat de la victime, les menaces ou le chantage.

\section{E ugénie: les fusils}

«Q uelques agresseurs sexuels achètent la coopération ou le silence de leur vidime avec de l'argent ou des faveurs.»

\section{L'agresseur d'E ugénie (8 ans) avait de nombreux fusils qu'il manipulait devant ses victimes \\ $L$ 'agresseur $n$ 'a pas besoin de verbaliser le rapport que les fusils peuvent avoir avec le fait de parler. L'en- fant va établir la connexion de lui-même.}

Q uelques agresseurs sexuels achètent la coopération ou le silence de leur victime avec de l'argent ou des faveurs. C es objets 
peuvent être proposés avant que la victimisation ne prenne place. L'acceptation de l'arrangement est particulièrement culpabilisante pour la victime, puisqu'elle a l'impression d'avoir accepté la proposition de son plein gré, même lorsqu'en fait, elle n'avait pas le choix, qu'elle en ait été consciente ou non. D ans d'autres situations, le don est fait après coup, comme une récompense, un paiement ou une compensation.

\section{N orma: le chèque}

$\mathrm{N}$ orma a 13 ans Elle témoigne en cour contre son frère $C$ 'est le procureur qui l'interroge: «0 ù vous at-il emmenée?»

- En bas.

- Et vous dites qu'il vous a «baisée»?

-0 ui.

- B on. A -t-il fait autre chose après ça?

- II m'a donné un chèque

- Bon. E t vous a-t-il dit pourquoi il vous donnait ce chèque?

- Pour que je ne le rapporte pas

- Pour que vous ne le disiez à personne?

- 0 ui.»

Bien que $\mathrm{N}$ orma soit handicapée mentale, elle a très bien saisi le lien qui a été établi par son frère entre le chèque et son silence $\mathrm{C}$ omme le chèque a été regu après le viol, l'enfant ne se sent pas culpabilisée.

\section{Crainte des réactions de l'agresseur}

$M$ ême lorsque l'agresseur sexuel n'utilise ni menace, ni promesse, l'enfant peut garder le silence par peur. D ansce cas, il imagine, à tort ou à raison, les conséquences possibles qu'aurait une révélation.

\section{F rançoise}

A u cours d'une enquête policière, F rançoise (8 ans) ré pond de cette façon non ambiguë à la question suivante: 
«Souvent l'enfant ne veut pas parler de ce qu'il a vécu car cela lui est trop pénible»
«Pourquoi n'en as-tu pas parlé à ta mère.. de ce qui se passait avec [nom de l'agresseur sexuel]?

- J'ai pensé qu'il serait en colère contre moi.»

L'enfant perçoit une partie de la réalité, ce que serait la réacion immédiate de son agresseur si elle parlait. E llen'est pas capabled' en imaginer les conséquences à long terme. E lle est prisonnière du futur immédiat.

\section{Qualité insupportable des souvenirs du traumatisme}

Souvent l'enfant ne veut pas parler de ce qu'il a vécu car cela lui est trop pénible. $0 \mathrm{n}$ va alors observer un arrêt dans son récit, une période de silence, parfois accompagnée de pleurs.

\section{G ermaine}

$G$ ermaine (12 ans) est interrogée par la police à propos d'incidents de maltraitance sexuelle qui se sont produits lorsqu' elle était toute petite. E lle a des souve nirs extrêmement clairs et préds. L ors des premiers interrogatoires, elle ne peut parler de ses expériences. Sa mère doit intervenir pour fadiliter la déposition. $M$ algré cela, à un moment donné, I'enfant reste silencieuse et ne peut élaborer plus avant.

$D$ ans œe cas, œ $n$ 'est pas l'insuffisance du langage ou des souvenirs qui cause le silence; c'est le poids émotionnel rattaché aux souvenirs.

\section{Raisons liées au milieu de soutien}

Beaucoup d'enfants gardent le silence sur leur maltraitance sexuelle pour des raisons qui tiennent à leur milieu de soutien, plutôt qu'aux caractéristiques de la maltraitance sexuelle ou à leur psychologie. C'est la perception qu'a l'enfant de son milieu qui compte, et non la réalité telle qu'elle serait évaluée par d'autres personnes. C'est de cette perception que va découler l'attitude de l'enfant. 
$M$ ême si l'enfant se sait aimé de ses parents, et même si aucun des autres facteurs énumérés jusqu'à présent n'a de grande importance, il peut ne pas dévoiler l'abus.

D eux types de raisons entrent en jeu. D 'une part, les facteurs développementaux sont importants. U n enfant évalue la qualité de son système de soutien en fonction de critères qui peuvent différer considérablement de ceux des adultes. De facon plus précise, on peut dire que l'enfant va commettre des erreurs de jugement. II n'est pas capable d'évaluer à leur juste valeur d'une part, l'importance des épisodes traumatiques, et de l'autre, celle des motifsqui le poussent à garder le silence vis à-vis de ses parents. Communément, par exemple, il surestime l'aspect moral de la question et sous- estime l'amour de ses parents ou la gravité de sa victimisation. L'erreur de l'enfant provient de la nécessité dans laquelle se trouvent les parents de l'éduquer, alors qu'il n'est pas encore capable d'en juger le bien-fondé. L'enfant est donc plus conscient de l'aspect disciplinaire que de l'aspect affectueux de la relation.

D 'autre part, des facteurs probabilistes existent: I'enfant doit prédire à l'avance quelle sera la réaction de ses parents. C eux-ci peuvent être aimants et pleins de sollicitude et cependant réagir de façon exagérée lors de l'annonce de victimisation sexuelle. L'enfant, lorsqu'il anticipe une telle réaction, est rationnel, même lorsqu'il se trompe.

M ême si l'enfant est entouré de soins de bonne qualité, si préalablement aux épisodes de maltraitance sexuelle, il existait de graves problèmes dans la famille comme l'alcoolisme, la dissension, ou la maladie, le silence est fréquent. L'enfant veut ne pas ajouter

«L orsque l' enfant reçoit peu de soins ou de soutien de sa famille, il ne parle que rarement des expériences qu'il vit... » de stressà un système déjà en équilibre précaire (D amon et al. , 1990).

Lorsque l'enfant reçoit peu de soins ou de soutien de sa famille, il ne parle que rarement des expériences qu'il vit, maltraitance et autres. D ans ce cas, son attitude est basée sur une perception généralement réaliste (à savoir qu'il ne sera pas cru ou soutenu).

En conclusion, on peut dire que les qualités du milieu de soutien n'entrent en jeu que de façon indirecte, à travers la 
perception qu'en a l'enfant et en fonction des conclusions qu'il tire sur ce que sera la réaction de ce milieu.

L'insistance sur la différence entre les qualités objectives du milieu de soutien de l'enfant et la perception que celui-ci en a est justifiée. En effet, bien des parents pensent que si l'enfant ne leur a pas parlé, c'est parce qu'ils doivent être de mauvais parents, ou parce que l'enfant ne leur fait pas confiance, ou parce que l'enfant a mal fait. Parfois même, les parents ne vont pas croire à la réalité de la maltraitance sexuelle «puisque [l'enfant] ne m'en a pas parlé.»

Les illustrations suivantes montrent que l'enfant peut ne pas révéler sa maltraitance sexuelle en fonction de son milieu de soutien, entre autres, lorsqu'il craint la réaction de ses parents, Iorsqu'il pense qu'il sera rejeté ou puni, ou lorsqu'il pense qu'il ne sera pas cru.

\section{Henri}

A près le réit qu'il a fait de son exploitation sexuelle par son cousin de 16 anș H enri (11 ans, abusé à 8 ans) explique à la psychologue:

«J' avais peur de le direà la maison. J' avais peur qu'ils me jettent dehors de la maison. P eut-être ils pensent que je voulais le faire... ou parce que je l' ai fait. $N$ on, ils m'ont oru.»

L'enfant énumère deux raisons pour son silence: il a pensé qu'il serait rendu responsable de ce qui lui est arrivé, soit moralement («... je voulais le faire»), soit simplement pour avoir été impliqué ( «ou parce que je I'ai fait»). L'enfant distingue donc spontanément les deux sortes de responsabilité, facuelle et morale, dont nous avons parlé précédemment. II a pensé aussi que, peut-être, il ne serait pas cru.

II convient de noter quel'enfant ne dit pas qu'il est responsable, mais simplement que ses parents auraient pu le croire responsable. 
L'enfant n'a pas parlé à cause des réactions qu'il anticipait, réađions qui se sont révélées fausses $D$ ans son cas, les raisons de la levée du silence ont été son appartenance à une bonne famille, et aussi le fait qu'il souffrait de troubles importants de l'humeur, séquelles de la maltraitance sexuelle, et que ces troubles lui devenaient insupportables

\section{Isis: «Je ne veux pas lui dire»}

A 10 ans Isis a été molestée sexuellement par un jeune homme de 21 ans au cours d'une réunion de famille $\mathrm{Q}$ uelques années plus tard, une psychologue I'interroge à œ propos, au cours d'une évaluation:

«E $n$ as-tu parlé à quelqu'un?

- J'en ai parlé à mon amie (16 ans). E lle a dit: «O $n$ en parlera demain». E lle pensait que j'ima ginais des choses

- Tu penses qu' elle était gênée ou qu' elle ne te croyait pas?

- Elle m'a dit: «T 'es certaine? Tu fais pas des histoires?»

- E $n$ as-tu parlé à tes parents?

- Je vivais avec mon père J'étais très jeune Je ne pensais pas que mon père comprendrait.

- E t maintenant, tu penses qu'il aurait compris?

- $N$ on. Il est bien gentil et jel'aime beaucoup... mais non...

- Tu en as parlé à ta mère?

- Non.

- Pourquoi pas?

- Je ne veux pas lui dire Seul mon ami le sait. II m'a dit que c' était à moi (que c' était son droit de garder le secret).

- M ais cen'est pas à toi. C 'est I' homme qui a fait

ça.

- Je sais, mais je n' ai pas envie d'en parler à mes parents.» 
$0 \mathrm{n}$ peut faire plusieurs remarques à propos de ce dialogue $D$ ans le passé, I'enfant a parlé à son amie. E lle n'a pas parlé à son père avec qui elle vivait. E lle n'a pas parlé à sa mère non plus À présent, elle a parlé à son ami, mais ne veut toujours pas parler à ses parents.

C e qui a pu favoriser la révélation à l'amie, c'est leur participation commune à la même situation (la réunion de famille), la proximité (c' est cette amie qui se trouvait là à ce moment-là), et des facteurs d'identification (I'amie était de même sexe et un peu plus âgée; elle constituait donc une sorte de modèle en qui on pouvait se confier). D e la même façon, par la suite, elle en parle à son ami.

L'enfant explique clairement pourquoi elle n' a rien révélé à son père, sur le moment, plus tard et acuellement. II ne comprendrait pas Q ue cela soit une pré diction juste ou fausse importe peu: I' enfant ne se sent pas suffisamment proche de son père pour communiquer avec lui dans ce domaine L es rapports avec la mère étant encore plus distants qu' avec le père, la question de lui parler ne se pose même pas

$0 n$ doit ajouter que les remarques incrédules de l'amie d'I sis ont pu porter la vicime à supposer qu'on pourrait ne pas la croire, si elle parlait à d' autres personnes.

\section{Conclusion}

Le secret dans les cas de maltraitance sexuelle de l'enfant a été peu étudié jusqu'ici bien que ce soit un facteur reconnu depuis longtemps par les cliniciens. C ela tient à ce que la recherche actuelle préfère en majorité étudier ce qui est quantifiable plutôt qu'elle ne s'intéresse aux aspects qualitatifs et expérientiels. L'approche phénoménologique convient particulièrement bien à l'étude du secret en rassemblant des données qualitatives auprès des intéressés eux-mêmes. 
"U ne connaissance des différentes raisons pour lesquelles un enfant peut préfèrer garder le silence, .... est essentielle si l'on veut détecter les cas de maltraitance sexuelle... »
C e bref aperçu sur le silence des enfants dans les cas de maltraitance sexuelle permet de montrer la complexité de la question. Trois catégories de facteurs ont été isolées. La première série de facteurs se rapporte à l'enfant, soit en raison de sa personnalité propre, soit en tant qu'il se situe à un certain stade de développement, stade qui a une influence sur sa perception des choses. La seconde série de facteurs inclut tout ce qui touche l'agresseur et la maltraitance sexuelle vécue. $0 \mathrm{n}$ a tendance à sous estimer l'importance, qui est capitale, des facteurs de cette série. La troisième sér ie de facteurs comprend lesfacteursqui caractér isent le milieu de soutien de l'enfant et la perception qu'en a l'enfant.

U ne connaissance des différentes raisons pour lesquelles un enfant peut préfèrer garder le silence, au lieu que de révéler ses expériences, est essentielle si l'on veut détecter les cas de maltraitance sexuelle suffisamment tôt pour pouvoir la stopper. De plus, cette connaissance peut rendre d'autres problèmes moins énigmatiques. C'est ainsi que l'on comprend mieux pourquoi, même lorsqu'il appartient à une bonne famille, I'enfant peut choisir de ne pas parler. $0 \mathrm{n}$ peut donc mieux expliquer la réaction de leur enfant aux parents et, par contrecoup, permettre à ceux-ci d'envisager la possibilité de la maltraitance sexuelle.

\section{Notes}

1. Voici à ce sujet, les documents suivants: Bagley et al., 1991; B rassard et al., 1987; Brière, 1992; Browne et Finkelhor, 1986; Cicchetti et $C$ arlson, 1987; Finkelhor, 1979; Finkelhor et B rowne, 1985; Friedrich, 1990; G ouvernement du C anada, 1992, 1993; Kempe et Kempe, 1986; Pelsser, 1989; Sanford, 1980; Walker, Bonner et Kaufman, 1988; Walker, 1988; W illis et al., 1991a, 1991b.

2. Selon I'usage, le masculin sera utilisé dans son sens générique. II sera cependant fait exception à cette règle lorsque les victimes (de notre échantillon) sont exclusivement féminines (à l'adolescence).

3. Les prénoms des sujets sont fictifs. 
4. Pour les dialogues, nous avons utilisé des chevrons au début et à la fin du dialogue, et des tirets devant chaque changement d'interlocuteur.

\section{Bibliographie}

BAGLEY, C.R . et R .J.TH O M LISON (éd.) (1991). C hild sexual abuse: C ritical Perspedives on P revention, Intervention, and Treatment,Toronto, Wall et Emerson.

BAR AH AL, R . M . et al. (1981). «T he social cognitive development of abused children», Journal of Consulting and C linical Psychology, no 49, 508-516.

BELSKY, J. et J. VO N DR A (1989). L essons form child abuse: the determinants of parenting, 153-202 dans D. C icchetti etV. C arlson.C hild maltreatment:T heory and research on the causes and consequences of child abuse and neglect. C ambridge, Cambridge U niversity Press.

BOW M AN, E.S. et al. (1985). «M ultiple personality in adolescence: relationship to incestual experiences», Journal of the A merican A cademy of C hild Psychiatry, no 24, 109-114.

BR ADY K. (1979). F ather's days: A true Story of Incest, N ew York, D ell.

BR ASSAR D M .R .,R . GER M AIN et S.N . H ART (éd.) (1987). P sychological M altreatment of C hildren andY outh, $N$ ew York, Pergamon.

BR IER E, J.N . (1992). C hild A buseT rauma:T heory and T reatment of the lasting E ffects N ewbury Park, Sage.

BRO U SSAR D, S.D. et W.G.W AGN ER (1988). «C hild sexual abuse: W ho is to blame?», C hild A buse and $N$ egled, 12, 563-569.

BR OW N E,A. et D. FIN KELH O R (1986). « mpact of child sexual abuse:A review of the research», Psychological Bulletin, no 99,66-77.

CHU, J.A. et D. L. DILL. (1990). «Dissociative symptoms in relation to childhood physical and sexual abuse», A merican Journal of Psychiatry, no 147, 887-892.

CIC CHETTI D. (1989). «H ow research on child maltreatment has informed the study of child development: perspectives from developmental psychopathology», 377-431 dans D. CIC C H ET T I et V. CAR LSO N. C hild maltreatment: T heory and research on the causes and consequences of child abuse and neglect. $C$ ambridge, $C$ ambridge U niversity Press.

CICCHETTI, D. et M. BEEGHLY. (1987). «Symbolic development in maltreated youngsters: an organizano tional perspective», N ew D irections for C hild D eveloment, 36, 47-68.

CICCHETTI, D. et V. CAR LSON (1989). C hild maltreatment: Theory and resarch on the causes and consequences of child abuse and negled, $C$ ambridge, $C$ ambridge $U$ niversity Press.

DALDIN , H . (1988). «T he fate of the sexually abused child», C linical Sodial W ork Journal, no 16, 22-32.

DAM O N , L.L., J.A. CAR D et J.T O DD (1992). «ncest in young children», p. 148-172. dans R .T.

AM M ER M AN ,et M . HER SEN, (éd.) A ssessment of F amilyV iolence:A dinical and legal Sourcebook, $\mathrm{N}$ ew York, W iley.

DELL, P.F. (1989). «V iolence and the systemic view: The problem of power», F amily Process, no 28, 1-14.

DEN ZIN , N .K. (1986). «Postmodern social theory», Sociological T heory, no 4, 194-204. 
DEN ZIN , N .K. (1989). Interpretive B iography, N ewbury Park, C a., Sage.

FAR R ELL, L.T. (1988). «Factors that affect a victim's self-disclosure in father-daughter incest», C hild W elfare, no 67, 462-468.

FER GUSO N , H .B.et S. M EN DELSO N -AGES. (1991). «Evaluating child sexual abuse prevention programs», dans C.R . Bagley, et R .J. Thomlison (éd.), C hild sexual abuse: C ritical Perspedives on P revention, Intervention, and T reatment, Toronto, Wall et Emerson.

FIN KELH O R, D. (1979). Sexually vidimized children. R iverside, N ew York, Free Press.

FIN KELHOR, D. et A. BROWNE. (1985). «T he traumatic impact of child sexual abuse: a conceptualization», A merican Journal of 0 rthopsychiatry, no 55, 530-541.

FR IED R ICH ,W.N . (1990). P sychotherapy of Sexually A bused C hildren and their Families N ew York, $\mathrm{N}$ orton.

FRU DE, N . (1982). «T he Sexual N ature of Sexual A buse», C hild A buse and N eglect, no 6, 211-223.

GELIN AS, D.J. (1983) «T he persisting negative effects of incest», P sychiatry, no 46.

GEN TLES, I. et E. C ASSIDY (1991). «Evaluating the evaluators: Child sexual abuse prevention Do we know it works?», dans C.R . Bagley, et R .J.Thomlison, éd. C hild sexual abuse: C ritical Perspedives on Prevention, Intervention, and Treatment, Toronto, Wall et Emerson.

GO M ES-SCHWARTZ, B., J.M . HO RO W ITZ etA.P.CAR DAR ELLI. (1990). C hild Sexual A buse: The Initial E ffects, $N$ ew berry Park, C a.,Sage.

GOU VER NEM ENT DU CAN ADA (1992). R éponse fédérale au rapport: À la recherche de solutions. $\mathrm{R}$ apport du conseiller spécial en matière d'agressions sex uelles contre les enfants, $\mathrm{O}$ ttawa, $\mathrm{M}$ inistère des A pprovisionnements et Services $C$ anada.

GO U VER N EM EN T DU CAN ADA (1993). R apport d'étape sur la réponse du gouvernement fédéral: À la recherche de solutions. $R$ apport du conseiller spédial en matière d'agressions sexuelles contre les enfants, $O$ ttawa, $M$ inistère des A pprovisionnements et Services $C$ anada.

HER M AN , J. (1981). «Father-D aughter Incest», P rofessional Psychology, no 12, 76-80.

KEM PE, R .S. et C.H . KEM PE (1986). T he common secret: Sexual A buse of C hildren and A dolescents, $\mathrm{N}$ ew York, Freeman.

KLEIN P.et M.R .W EST C OTT (1994). «T he changing character of phenomenological psychology», $C$ anadian P sychology/ Psychologie canadienne, no 35, 133-158.

LOFTU S, E. (1993). «T he reality of repressed memories», A merican Psychologist, no 48, 518-537.

M CEVOY, M ., et J. DAN ILUK (1995). «Wounds to the soul :T he experiences of A boriginal women survivors of sexual abuse»,C anadian Psychology/ Psychologie canadienne, no 36, 221-235.

PELSSER , R . (1989). «Les abus physiques et sexuels», dans M anuel de psychopathologie de l'enfant et de I'A dolescent, M ontréal, Gaëtan M orin.

SAN FO R D, L.T. (1980). T he silent C hildren: A Parent's $G$ uide to the Prevention of $C$ hild Sexual $A$ buse, $\mathrm{N}$ ew York, M CG raw-Hill.

SCHULTZ, L.G. (1979). «The Sexual Abuse of Children and M inors: A Bibiliography»,C hild Welfare, no 58, 147-163.

SO R EN SEN , T. et B. SN OW (1991). «H ow children tell :T he process of disclosure in child sexual abuse», C hild-W elfare, no 70, 3-15.

WALKER, C.E., B.L. BONNER et K.L. KAUFMAN (1988). The physically and sexually abused child : E valuation and Treatment, $N$ ew York, Pergamon. 
WALKER , L.E.A. (1988). H andbook on sexual A buse of C hildren: A ssessment and Treatment I ssues, $\mathrm{N}$ ew York, Springer.

W ILLIS, D.J., W. BAGWELL et M.M. CAM PBELL (éd.) (1991a). C hild A buse: A bstracts of the Psychological and Behavioral Literature. Vol. 1, 1967-1985, Washington, American Psychological Association.

W ILLIS, D.J., G.C. BR OY HILL et M .M. CAM PBELL (éd.) (1991b). C hild A buse: A bstrads of the Psychological and Behavioral Literature. Vol. 2, 1986-1990, Washington, American Psychological Association. 